COMPUTATIONAL METHODS IN SCIENCE AND TECHNOLOGY 8(2), 65-76 (2002)

\title{
ADVANCED METHODS OF HEART RATE SIGNALS PROCESSING AND THEIR USEFULNESS IN DIAGNOSIS SUPPORT. I. MATHEMATICAL HEART RATE DESCRIPTORS AND VIRTUAL INSTRUMENTATION
}

\author{
JERZY A. MOCZKO \\ Chair and Department of Computer Science and Statistics, \\ University of Medical Sciences in Poznań, Dabrowskiego 79, 60-529 Poznań, Poland, \\ e-mail: jimoczko@am.poznan.pl
}

\begin{abstract}
The working heart is the source of electrical signal which carries the basic information about homeostasis process of human being. After appropriate acquisition and preprocessing electrocardiogram (ECG) signal may be analyzed in different ways revealing not only current status of atrial and ventricular electrical activity of heart muscle but also information about sympathetic and parasympathetic nervous system, thermoregulation and vasomotor tone, renin-angiotensin control systems, baroreceptor reflex and blood pressure regulation, respiratory activity etc. Heart rate variability (HRV) defined as a timing of the sequence of QRS complexes is one of the most promising quantitative markers of autonomic activity. Different mathematical indices in time and frequency domain were designed to extract full details valuable for diagnostic purposes. Presented paper is the first one of the series of articles describing mathematical methodology of heart rate (HR) signal processing and further classification of obtained markers. The ultimate goal is to find optimal set of HR descriptors which support diagnosis in different cardiac pathologies.
\end{abstract}

\section{INTRODUCTION}

Electrocardiogram is one of best known and deeply analysed biomedical signals. Direct approach based on examination of changes in the temporal durations of the segments between deflections, intervals between waves, their relative amplitudes is the essential way of interpretation of the ECG wave patterns. A wide spectrum of various mathematical methods in time, frequency and joined time-frequency domains are used to extract most distinctive features of the ECG which may be used as the markers in different pathologies. These markers should have not only the best sensitivity and specificity, but they should be maximally noninvasive, readily available and inexpensive.

The intensive research proved the existence of significant relationship between the autonomic nervous system and cardiovascular mortality. Heart rate variability (HRV) estimated as the recurrent changes in $R-R$ intervals can be good prognostic indicator for large amount of different disease states. Decreased HRV is a powerful predictor of arrhythmic complications and cardiac death. It is treated as a versatile test to determine risk stratification and supports the choice of treatment strategies. Tens of different HRV indices vere designed for assessing the integrity of cardiovascular homeostasis. Some of them (i.e. SDNN, RMSSD, HRV triangular index in time domain 
or LF, HF, LF/HF ratio in frequency domain) are most frequently used in clinical practice due to relatively simple interpretation and good prognostic properties. Comprehensive review of these indices prepared as task force of the European Society of Cardiology and the North American Society of Pacing and Electrophysiology may be found in [1], Teich et al. [2] examined suitability for correctly classifying ECG records as normal or representing the presence of cardiac dysfunction on the basis of sixteen different HRV measures. These papers were the inspiration for further investigations on HRV indices properties, which we performed in cooperation with Cardiology Department of our university.

Presented paper is the first one of the series presenting different mathematical approaches to diagnosis support. We present virtual instrumentation designed for ECG signal acquisition and processing. This instrumentation is the source of the set of thirty six HRV descriptors in time, frequency and joined time-frequency domain estimated for normal or pathological ECG recordings. The obtained database allows for checking the suitability of particular indices for discrimination patients with different cardiac pathologies from normal subjects.

The difference between Teich et al. and our results yields from the fact of using multivariate instead of univariate approach. Various classification methods (multivariate statistical techniques such as general discriminant analysis, classification and regression trees, artificial neural networks, generation of relevant fuzzy rules) and their usefullness in diagnosis support is discussed.

\section{HEART RATE VARIABILITY INDICES}

The mathematical structure of most known HRV indices is based on statistical parameters such as arithmetic mean value, standard deviation, interquartile range. Some of them are estimated directly from $R-R$ intervals, the other incorporate heart rate (HR) values calculated with hyperbolic dependence

$$
\mathrm{HR}=\frac{60000}{T_{R-R}}
$$

where $T_{R \_R}$ is expressed in miliseconds, HR in beat per minutes (bpm). One can divide them into three subgroups:

1) long-term variability indices - which describe slow changes in HR (low frequencies),

2) short-term variability indices - describing changes in consecutive $R-R$ intervals (high frequencies),

3) total variability indices - taking into account all sources of variation (full band spectrum).

The group of time domain measures (not directly related to specific cycle length) include following indices:

1) SDNN index - is defined as standard deviation of subsequent $R-R$ intervals. This measure is sensitive to all sources of variation (both long- and short-term variability). 


$$
\mathrm{SDNN}=\sqrt{\frac{\sum_{i=1}^{N-1} T_{R R}(i)}{N-1}}
$$

2) RMSSD index (Root-Mean__Square of Successive-interval Differences) - is computed from triplets of normal beats ( $\mathrm{R}$ waves). Each triplet defines two included adjacent coupling intervals. The difference between the two coupling intervals is squared and summed. Finally such obtained sum is divided by number of intervals and square root is calculated. RMSSD is most sensitive to the highest frequency components (short term variability) of HR.

$$
\text { RMSSD }=\sqrt{\frac{\sum_{i=1}^{N-1}\left[T_{R R}(i)-T_{R R}(i-1)\right]^{2}}{N-1}}
$$

3) Yeh differential index DI - short term variability index - defined as the standard deviation of $d(i)$ ratios estimated for 30 second length signal ( $N$ - number of beats in 30 seconds) [3]

$$
\mathrm{DI}=\sqrt{\frac{1}{N-2} \sum_{i=1}^{N-1}(d(i)-\bar{d})^{2}}
$$

where

$$
d(i)=\frac{T_{R R}(i)-T_{R R}(i+1)}{T_{R R}(i)+T_{R R}(i+1)}
$$

and

$$
\bar{d}=\frac{1}{N-1} \sum_{i=1}^{N-1} d(i)
$$

4) Yeh interval index II - long term variability index - defined as coefficient of variability of subsequent $T_{R R}$ intervals estimated for 30 second window [3]

$$
\mathrm{II}=\frac{\sqrt{\frac{1}{N} \sum_{i=1}^{N}\left(T_{R R}(i)-\bar{T}\right)^{2}}}{\bar{T}}
$$

where

$$
\bar{T}=\frac{1}{N} \sum_{i=1}^{N} T_{R R}(i)
$$

5) De Haan STV index - short term variability index - defined as interquartile range of phase location of particular $T_{R R}$ intervals in two dimensional $\left[T_{R R}(i), T_{R R}(i+1)\right]$ space estimated for 128 consecutive $R$ waves [4] (Fig. 1) 


$$
\mathrm{STI}=\operatorname{IQR}\left(\varphi_{i}\right)
$$

where

$$
\varphi_{i}=\operatorname{atan}\left(\frac{T_{R R}(i)}{T_{R R}(i-1)}\right)
$$

6) De Haan LTV index - long term variability index - defined as interquartile range of radius location of particular $T_{R R}$ intervals in two dimensional $\left[T_{R R}(i), T_{R R}(i+1)\right]$ space estimated for 128 consecutive $R$ waves [4] (Fig. 1)

$$
\mathrm{LTI}=\operatorname{IQR}\left(r_{i}\right)
$$

where

$$
r_{i}=\sqrt{T_{R R}^{2}(i-1)+T_{R R}^{2}(i)}
$$

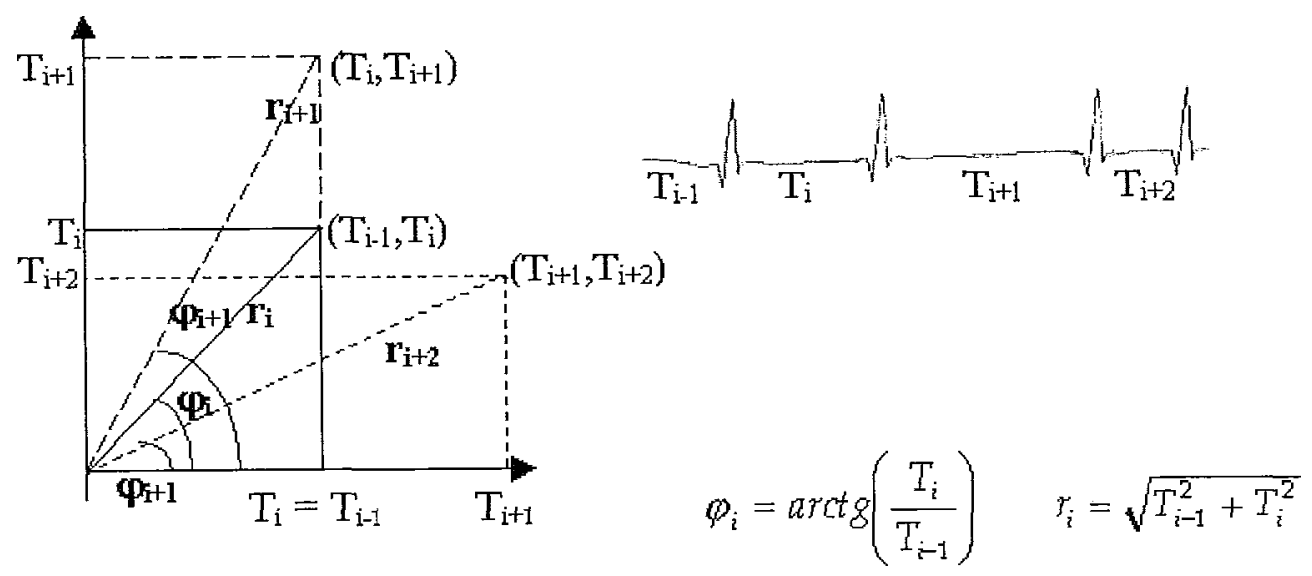

Fig. 1. Distribution of $T_{R R}$ intervals in two dimensional space $\left\langle T_{R R}(i), T_{R R}(i+1)\right\rangle$

7) Organ BAND index - long term variability index - defined as doubled standard deviation of HR sequence estimated in 30 second width window [5]

$$
\mathrm{BAND}=2 \cdot \sqrt{\frac{1}{N-1} \sum_{i=1}^{N}(\mathrm{HR}(i)-\overline{\mathrm{HR}})^{2}}
$$

where HR is arithmetic mean value of heart rate in analysed window.

8) van Geijn ID index - short term variability index - defined as interquartile range of weighted differences between successive $T_{R R}$ intervals estimated in 30 second window [6]

$$
\mathrm{ID}=\operatorname{IQR}\left\{g_{i}\left(T_{R R}(i)-T_{R R}(i-1)\right)\right\}
$$


where

$$
\begin{aligned}
& g_{i}=\left(\frac{180}{\bar{T}_{R R}(i)-320}\right)^{1.5} \\
& \overline{T_{R R}(i)}=\frac{T_{R R}(i)+T_{R R}(i-1)}{2}
\end{aligned}
$$

For $\bar{T}_{i}<381 \mathrm{~ms}$ gj is constant and equal to 5 .

9) Huey HSTV index - short term variability index - defined as a sum of modules (absolute values) of subsequent HR differences, for which change of the sign was observed in period equal to one hundred $T_{R R}$ intervals [7]

$$
\operatorname{HSTV}=\sum_{i=1}^{N-1} k \cdot|\mathrm{HR}(i+1)-\operatorname{HR}(i)|
$$

where

$$
k=\left\{\begin{array}{l}
1 \text { for }[\mathrm{HR}(i-1)-\mathrm{HR}(i)] \cdot[\mathrm{HR}(i)-\mathrm{HR}(i+1)]<0 \\
0 \text { for }[\mathrm{HR}(i-1)-\mathrm{HR}(i)] \cdot[\mathrm{HR}(i)-\mathrm{HR}(i+1)] \geq 0
\end{array}\right.
$$

10) Huey HLTV index - long term variability index - defined as a sum of modules (absolute values) of subsequent HR differences, for which change of the sign was not observed in at least three subsequent differences (in at least three heart beats we did not detect the change in monotonicity of HR signal) in period equal to one hundred $T_{R R}$ intervals [7]

$$
\mathrm{HLTV}=\sum_{i=2}^{N-1} l \cdot|\mathrm{HR}(i+1)-\mathrm{FHR}(i)|
$$

where

$$
l=\left\{\begin{array}{l}
1 \text { for }[\mathrm{HR}(i-2)-\mathrm{HR}(i-1)] \cdot[\mathrm{HR}(i-1)-\mathrm{HR}(i)] \cdot[\mathrm{HR}(i)-\mathrm{HR}(i+1)]>0 \\
0 \text { for }[\mathrm{HR}(i-2)-\mathrm{HR}(i-1)] \cdot[\operatorname{HR}(i-1)-\operatorname{HR}(i)] \cdot[\operatorname{HR}(i)-\operatorname{HR}(i+1)] \leq 0
\end{array}\right.
$$

11) Dalton MABB index - short term variability index - defined as one half of arithmetic mean value of absolute value of differences of subsequent $T_{R R}$ intervals estimated for 2 minute length HR signal [8]

$$
\operatorname{MABB}=\frac{1}{N} \sum_{i=1}^{N} \frac{\left|T_{R R}(i)-T_{R R}(i+1)\right|}{2}
$$

12) Dalton DSD index - long term variability index - defined as standard deviation of $T_{R R}$ estimated for 2 minute length HR signal [8]

$$
\mathrm{DSD}=\sqrt{\frac{1}{N-1} \sum_{i=1}^{N}\left(T_{R R}(i)-\overline{T_{R R}}\right)^{2}}
$$


where

$$
\bar{T}_{R R}=\frac{1}{N} \sum_{i=1}^{N} T_{R R}(i)
$$

and $N$ is equal to number of intervals in two minute length HR signal.

13) Zugaib ZSTV index - short term variability index - defined as averaged deviation estimated for 128 subsequent $T_{R R}$ intervals [9]

$$
\mathrm{STV}=\frac{1}{N-1} \sum_{i=1}^{N}|D(i)-M d[D(i)]|
$$

where

$$
D(i)=\frac{\left|T_{R R}(i+1)-T_{R R}(i)\right|}{T_{R R}(i+1)+T_{R R}(i)}
$$

and $M d[D(i)]$ is median value of 128 elements of $D(i)$ sequence.

14) Zugaib ZLTV index - long term variability index - defined as defined as averaged deviation estimated for 128 subsequent $T_{R R}$ intervals [9]

$$
\text { ZLTV }=\frac{1}{N} \sum_{i=1}^{N} \frac{\left|T_{R R}(i)-M d\left[T_{R R}(i)\right]\right|}{M d\left[T_{R R}(i)\right]}
$$

where $M d\left[T_{R R}(i)\right]$ is median value of $128 T_{R R}(i)$ intervals.

15) Oscillation index - sensitive to all sources of variation (both long- and short-term variability) - defined as a range of the heart rate measured during 60 seconds period in the area of signal's stationarity (absence of accelerations or decelerations)

$$
\operatorname{OSC}=\max (\operatorname{HR}(\mathrm{i}))-\min (\operatorname{HR}(\mathrm{i}))
$$

where

$$
i \quad=1 \ldots N
$$

$N$ - number of HR samples in 60 seconds interval.

Second analysed group of indices are these constructed in frequency domain. According to Sayer's model of heart rate variability [10] the existence of three main components in this domain was proved attributing physiologic origins as follows:

- Low-frequency component (0.02-0.09 Hz> - mediated by sympathetic and parasympathetic nervous system, renin-angiotensin control systems and thermoregulation mechanism;

- Mid-frequency component $(0.09-0.15 \mathrm{~Hz}>-$ resulting from the baroreceptor reflex and blood pressure regulation;

- High frequency (0.15-1.1 Hz> - respiratory band (parasympathetic).

In practice five heart rate variability indices are used [1]:

1) Ultra-low frequency range power ULF - frequencies $\langle\mathrm{DC}, 0.003 \mathrm{~Hz}\rangle$ - this band corresponds to periods greater than 333.3 seconds and is used in Holter records analysis. 
2) Very-low frequency range power VLF - frequencies $(0.003 \mathrm{~Hz}, 0.04 \mathrm{~Hz}>$ - this band corresponds to periods between 25.0-333.3 seconds and from physiological point of view it has not been specifically identified.

3) Low-frequency range power LF - frequencies $(0.04 \mathrm{~Hz}, 0.15 \mathrm{~Hz}>-$ this band corresponds to periods between 6.7-25.0 seconds and reflects both sympathetic and parasympathetic (vagal) activity.

4) High-frequency power $\mathrm{HF}$ - frequencies $(0.15 \mathrm{~Hz}, \mu \mathrm{HZ}>$ - this band corresponds to periods lower than 6.7 seconds and its upper limit is different for adults $(\mu=0.4 \mathrm{~Hz})$, neonates $(\mu=0.8 \mathrm{~Hz})$ and fetuses $(\mu=1.1 \mathrm{~Hz})$.

5) The ratio of the power in LF band to that in HF range LF/HF - this index is treated as the marker of sympathetic modulation (sympathovagal balance).

In presented paper we used two methods of power spectrum estimation: nonparametric based on Fast Fourier Transform and parametric based on covariance autoregressive modeling model order equal to twelve. The power in particular bands is not represented as absolute power but jt is normalized to total power of the signal $<0$, Nyquist frequency $>$.

For parametric spectrum estimation we obtain additionaly roots locations (complex values) and filter coefficients. The number of these parameters depends on model order (value 12 was used as default).

The last group of indices are scale-dependent measures.

1) Allan Factor $A(T)$ - is the ratio of the Allan variance of a sequence of counts $N_{i}(T)$ in a predetermined time interval $T$ (in other words number of $R$ waves that fall in the $i$-th window of duration $T$ seconds) to twice the mean [2]

$$
A(T)=\frac{E\left\{\left[N_{i+1}(T)-N_{i}(T)\right]^{2}\right\}}{2 E\left\{N_{i+1}(T)\right\}}
$$

where $E\{$.$\} represents expectation operator (for normal didtribution equal to arithmetic mean value).$

The derivative structure of the Allan factor allows to avoid problems with linear nonstationarities in analysed signal.

2) The wavelet-transform standard deviation [2]

$$
\sigma_{w a v}(m)=\left\{E\left[\left|W_{m, n}(m)\right|^{2}\right]\right\}^{1 / 2}
$$

where E\{.\} represents expectation operator and dyadic discrete wavelet transform for the $T_{R R}\{i$ ) sequence is defined as

$$
W_{m, n}(m)=\frac{1}{\sqrt{m}} \sum_{i=1}^{N} T_{R R}(i) \psi\left(\frac{i-1}{m}-n\right)
$$

$N$ - is a number of $T_{R R}$ intervals, $m$ - scale factor (related to the scale index $j$ by relation $m=2^{j}, n$ - is translation value and $\psi$ is wavelet basis function.

Taking Haar-wavelet as a basis wavelet and scale factor $m=1$ we obtain the result identical to RMSSD index. The wavelet-transform standard deviation is a marker constructed in joined time- 
frequency domain which describes signal properties at all scales. Typically its value increases with the scale $m$. Similarly as in Allan factor the trends and baseline variations are removed by the transform.

\section{NORMALIZATION OF ANALYSED INDICES AND POWER SPECTRUM ESTIMATION}

The definitions of the indices described in previous chapter reveal the fact that direct comparison of their properties is impossible due to different time window width. In Table I window width and corresponding number of RR intervals (calculated assuming average heart rate equal to 72 beats per minute $(\mathrm{bpm})$ ) vere gathered. To avoid this problem in designed virtual instrument (described in details in the next chapter) two options of indices are available:

1) original - estimating index value strictly according to the definition;

2) one-minute width - estimating index value in normalized one-minute width window.

Such a normalization results in slight changes of Yeh, de Haan, Organ, van Geijn, Huey, Zugaib, Dalton and oscillation indices and does not affect the others. The changes vere constant in research because the length of HR signal was equal to one minute.

Table I. Comparison of window wirth and corresponding number of $T_{R R}$ intervals in chosen HRV indices calculated for the average HR level equal to $72 \mathrm{bpm}$

\begin{tabular}{lccc}
\hline HRV Index & Definition & $\begin{array}{c}\text { No. } \\
\text { of corresponding } T_{R R}\end{array}$ & $\begin{array}{c}\text { No. } \\
\text { of seconds }\end{array}$ \\
\hline Yeh & $30 \mathrm{sec}$ window & 36 & $\mathbf{3 0}$ \\
De Haan & 128 beats & $\mathbf{1 2 7}$ & 106 \\
Organ & $30 \mathrm{sec}$ window & 36 & $\mathbf{3 0}$ \\
Van Geijn & $30 \mathrm{sec}$ window & 36 & $\mathbf{3 0}$ \\
Huey & $100 R R$ intervals & $\mathbf{1 0 0}$ & 83 \\
Zugaib & $128 R R$ intervals & $\mathbf{1 2 8}$ & 106 \\
Dalton & $120 \mathrm{sec}$ window & 100 & $\mathbf{1 2 0}$ \\
Oscillation & $60 \mathrm{sec}$ window & 72 & $\mathbf{6 0}$ \\
\hline
\end{tabular}

The indices we used in analysis are not all possible but most known and frequently used measures of HRV estimation.

The second analysis problem results from the fact, that heart rate signal does not represent typical time series but time event. There exist a few approaches of time events power spectrum (i.e. Lomb spectrum for nonequidistant samples, spectrum of counts, interval spectrum) [2, 11, 12]. In presented paper Berger equidistant resampling of HR signal was used [13]. 


\section{VIRTUAL INSTRUMENTATION}

\section{AND DETAILED PROGRAM DESCRIPTION}

LabVIEW (Laboratory Virtual Instrumentation Engineering Workbench - ${ }^{\circledR}$ National Instruments Corporation, Austin, Texas, US) is a graphical programming platform that has been widely adopted throughout research laboratories, universities and industry for data acquisition and processing. Contrary to classical (text-based) programming languages such as C, FORTRAN, PASCAL this environment is based on the concept of data flow programming. Instead of creating lines of code programmer creates programs in pictorial form (block diagram). The elimination of a lot of syntactical details allows to concentrate on substantial part of resolving problem. LabVIEW relies on graphical symbols rather then textual representation of operations, variables, functions, procedures etc.

The main principle of dataflow states that the element in block diagram executes only after receiving the necessary data. Programs prepared in LabVIEW are called virtual instruments becaurse their appearance and operation resemble actual instruments. On front panel different knobs, push buttons, slides and other controls or chart, graphs, displays and other indicators are available. Virtual instruments (VI) are hierarchical and modular. One can use them either as top-level (main) programs or subprograms. Each VI is represented pictorially. The data flow between different level procedures is realized with connectors, which define the inputs and outputs of the VI similarly as parameters in a subroutine do it.

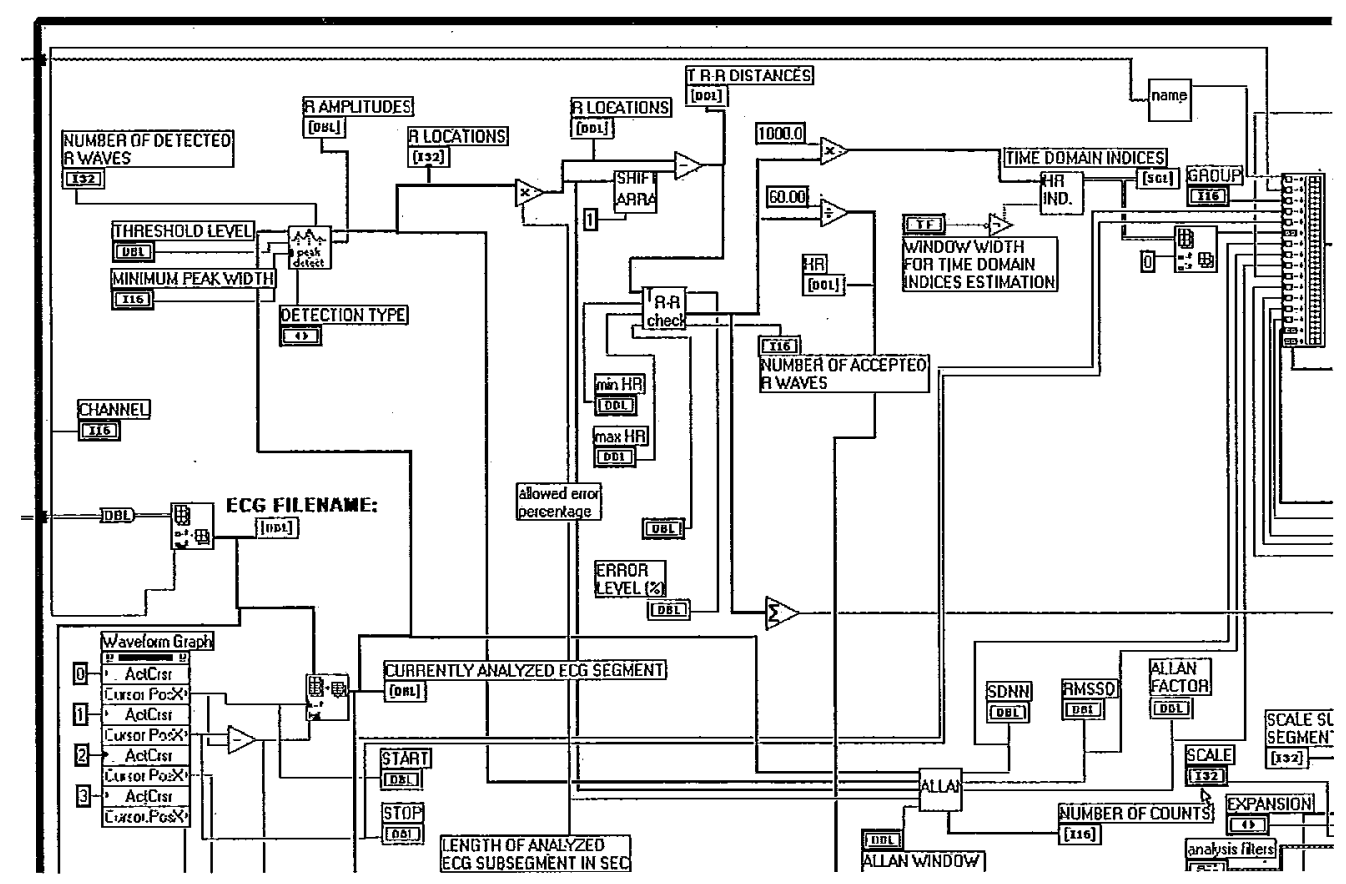

Fig. 2. Fragment of block diagram of VI for heart rate variability analysis 
Lab VIEW is not only a powerful calculation tool, but it offers possibility of gathering data from external sources. It facilitates data transfer over the paralel GPIB or serial RS ports. Data Acquisition (DAQ) cards directly controlled by appropriated VI-s allow to acquire or generate digital and analog signals. A VI library with great number of digital signal processing procedures and INTERNET technologies support make Lab VIEW the ideal tool to design program which allow perform complex analysis of heart rate variability $[14,15]$. Figure 2 presents fragment of block diagram of presented program.

Organization of the research work is as follows:

1) In Cardiology Department of University of Medical Sciences 5 minute length 12 channels ECG recordings of patients with different cardiac pathologies (more medical details would be presented in next article) and control group of healthy people are gathered with KARDIOGRAF KARDIO PC (® MEDEA - Medical Equipment Advance. Gliwice, Poland) with sampling frequency $500 \mathrm{~Hz}$. The amplitude resolution of Analog-to-Digital Converter is 12 bits. All preprocessing procedures correspond to American Heart Association norm for analog filtration. The data are saved in binary files.

2) Data records are transferred to Department of Computer Science via Internet using FTP protocol.

3) The analysis of ECG tracings is performed with specialy designed VI and the results are saved in database (simple ASCII file).

4) Final data are analyzed with different statistical and data-mining techniques such as general discriminant analysis, logistic regression, ROC - area analysis, classification and regression trees, fuzzy rules etc. The results of this analysis and comparison of usability of mentioned methods we present in next paper.

ECG signal may be acquired either form binary format (binary format consistent with KARDIOGRAF KARDIO PC) or from ASCII files. Operator may choose channel number and mode of detection (peaks or valleys dependently on the channel type). In next step the subsegment of artifact free ECG tracing is cut off interactively using vertical cursors. To detect $\mathrm{R}$ waves original PEAK DETECTOR. VI (TIME DOMAIN Lab VIEW library) was used. Operator changes with slides threshold level and minimum peak width observing simultaneously ERROR LEVEL and NUMBER OF ACCEPTED R WAVES indicators. Two X-Y type graphs display respectively the analysed ECG segment and heart rate curves before and after Berger filtration (Fig. 3). The chosen ECG segment, corresponding HR record and estimated HRV indices may be saved in ASCII file by pressing appropriate push buttons.

The results of ECG analysis are displayed on indicators in the lower part of VI. Front panel reports number of detected $R$ waves, $R$ locations (as a sample number and in seconds), $R$ waves amplitudes, $T_{R R}$ intervals and number of counts in Allan window (default - 2 seconds). Heart rate section displays HR values before Berger filtration and allowed percentage of erroneous samples (the admissible range of heart rate values is defined by specifying minimum and maximum HR value). Four knobs allow to change the parametrs of Berger filtration. With horizontal toggle switch one changes the way in which HRV indices are calculated (Fig. 4). 


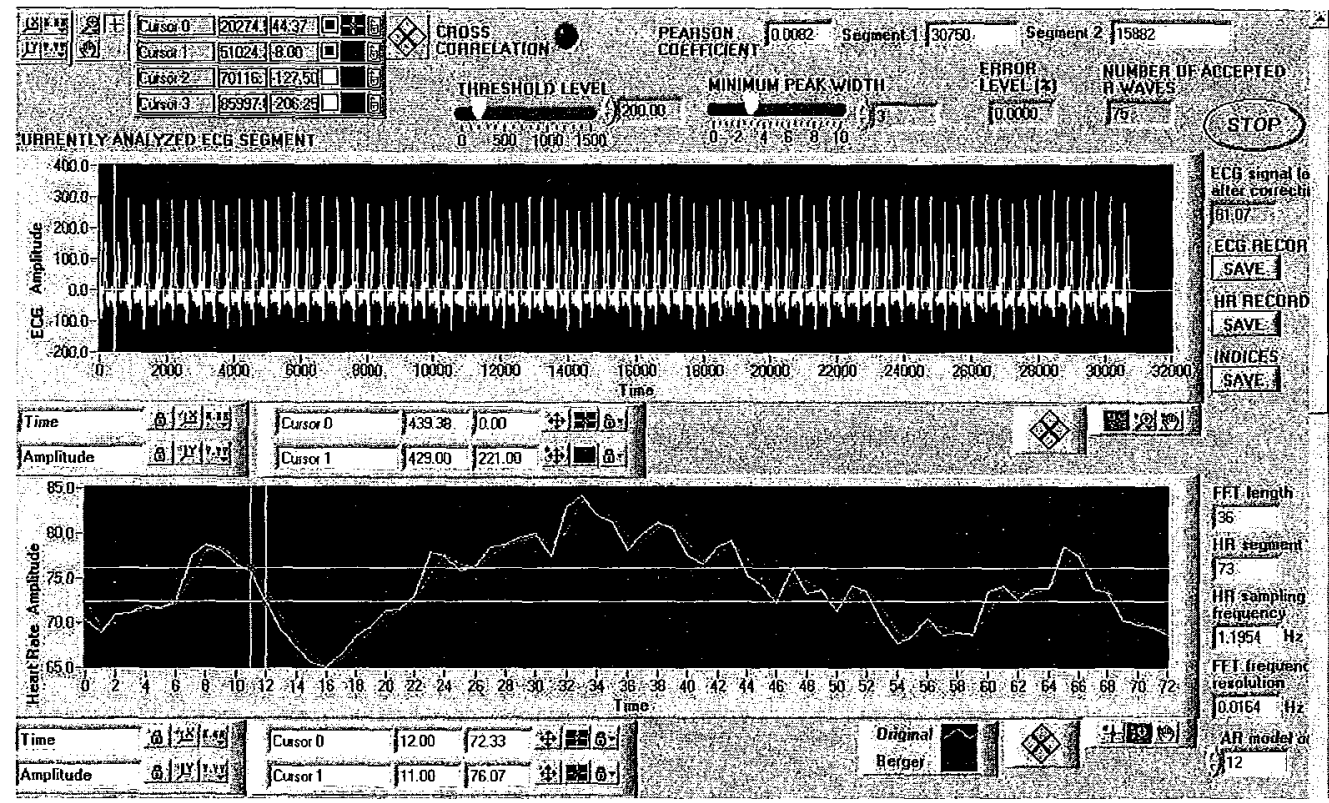

Fig. 3. ECG subsegment choice and its preprocessing

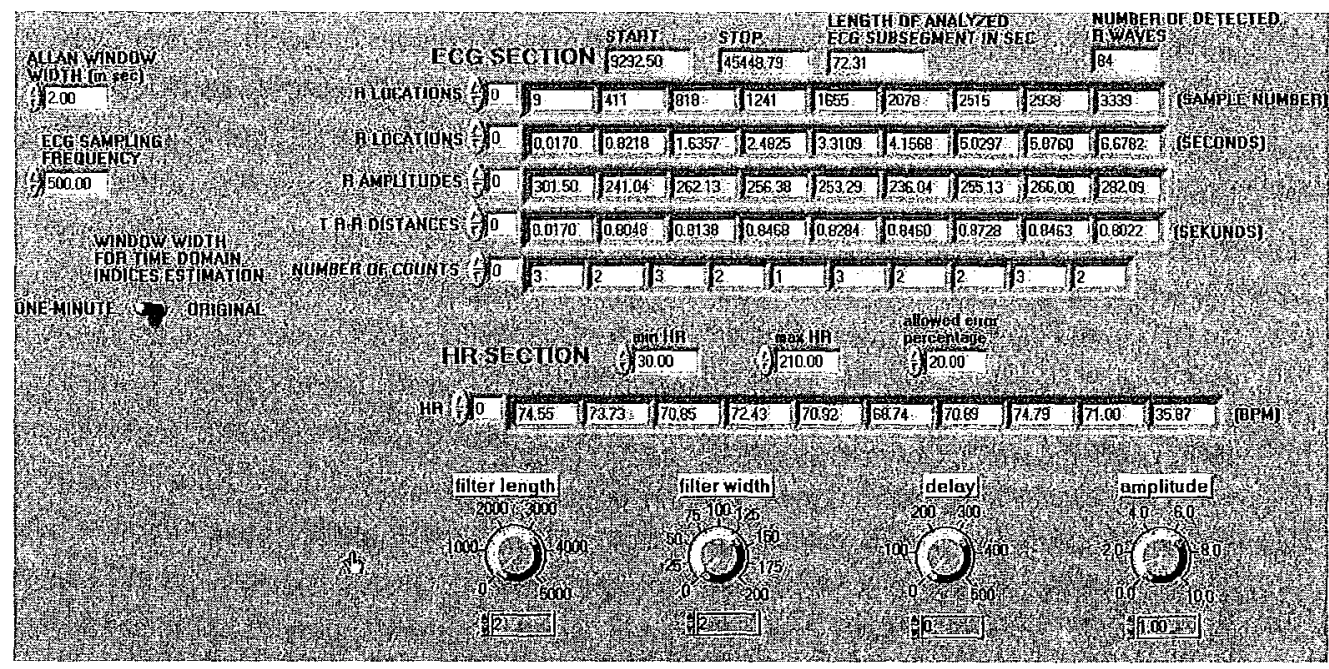

Fig. 4. $R$ waves detection results and HR estimation

The results of analysis are displayed in appropriate indicators (Fig. 5). When operator is sure the quality of analysed signal is good and all controls are properly ajusted, he may save the final results in append mode in ASCII database, which is the source of information for further multidimensional statistical and data-mining analysis. The ultimate goal of this analysis is not comparison of mathe- 


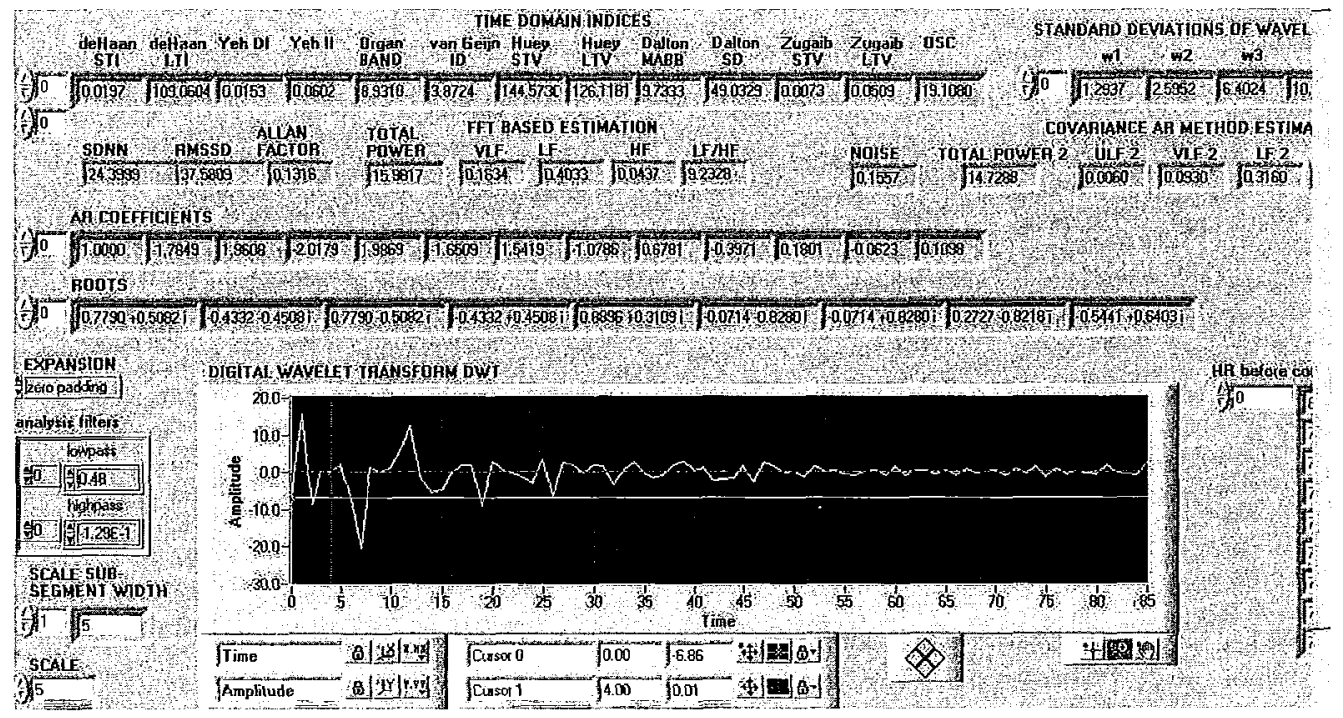

Fig. 5. Fragment of front panel with HRV indices

matical properties of described measures but an attempt to answer the question if multivariate approach to HRV analysis may improve quality of diagnosis support. The definitions quoted in chapter 2 reveal the fact, that each measure takes into account only some part of information carried by ECG signal.

\section{References}

[1] Eur. Heart J., 17, 354-381 (1996).

[2] M. C. Teich et al,, IEEE Press Series Biomed. Eng., NY, 159-213 (2001).

[3] S. Y. Yeh, A. Forsythe, E. H. Hon, Obstet. Gynecol., 41, 355-363 (1973).

[4] 1. de Haan et al., Europ. I. Obstet. Gynec., 4, 137-146 (1971).

[5] L. W. Organ et al., Am. J. Obstet. Gynec., 130, 20-27 (1978).

[6] H. P. van Geijn, Am. J. Obstet. Gynec., 138, 246-252 (1980).

[7] J. R. Huey et al., Am. J. Obstet. Gynec., 134, 691-695 (1979).

[8] K. J. Dalton, G. S. Dawes, J. E. Patrick, Am. J. Obstet. Gynec., 127,414-424 (1977).

[9] M. Zugaib et al., Am. J. Obstet. Gynec., 138,444-452 (1980).

[10] B. McA. Sayers, Ergonomics, 16,17-32 (1973).

[11] R. W. DeBoer, J. M. Karemaker, J. Strackee, IEEE Trans. Biomed. Eng., BME-31, 4 (1984).

[12] J. Vila, IEEE Ann. Conf. Eng. in Med. Biol. Soc., 575-576 (1992).

[13] R. D. Berger, IEEE Trans, BME-33, 900-904 (1986).

[14] I. B. Olansen, E. Rosow, Virtual Bio-Instrumentation. Biomedical, Clinical and Healthcare Applications in LabVIEW. Prentice Hall PTR, (2002).

[15] M. L. Chugani, A. R. Sammant, M. Cerna, LabVIEW Signal Processing. Prentice Hall PTR (1998). 